\title{
Phosphorylation-dependent mitochondrial translocation of MAP4 is an early step in hypoxia-induced apoptosis in cardiomyocytes
}

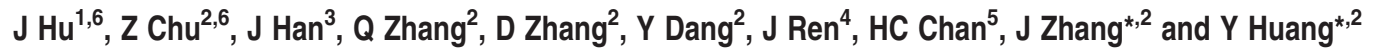

Hypoxic or ischemic apoptosis is often tightly associated with the opening of mitochondrial permeability transition pore (mPTP); however, the molecular mechanisms regulating mPTP and thus mitochondrial-dependent apoptosis remain elusive. Emerging evidence indicates that the movement of key proteins in or out of mitochondria play a critical regulatory role in apoptosis. Here, we reported that, unexpectedly, the microtubule-associated protein 4 (MAP4) translocated from cytosol to mitochondria upon phosphorylation after hypoxia treatment in neonatal cardiomyocytes. When targeted to mitochondria, MAP4 was found to lead to MPTP opening and induce apoptosis. Mitochondrial accumulation and pro-apoptotic function of MAP4 could be reversed through the genetic inhibition of MAP4 phosphorylation. The MAP4(Ala) mutant, which mimicked the dephosphorylated form, suppressed mitochondrial translocation and apoptosis. Our data reveal a novel role of MAP4 in cardiac apoptosis and suggest a potential therapeutic strategy targeting mitochondrial translocation of MAP4 against apoptotic heart diseases.

Cell Death and Disease (2014) 5, e1424; doi:10.1038/cddis.2014.369; published online 18 September 2014

Cardiovascular disease remains the leading cause of death worldwide, accounting for $30 \%$ of all global deaths. ${ }^{1-3}$ Myocardial ischemia, and thereby inadequate supply of oxygen and nutrients to the heart, mediates cardiac dysfunction and death. The evidence appears to support the argument that apoptosis or programmed cell suicide contributes to the initiation and progression of cardiomyocyte loss in ischemia conditions such as heart failure and myocardial infarction that results in contractile tissue loss, compensatory hypertrophy and reparative fibrosis and ultimately contributes to the development of cardiac dysfunction or even sudden death. ${ }^{4,5}$ Better understanding of the mechanisms underlying cardiomyocyte apoptosis may therefore hopefully yield novel therapeutic targets against these cardiac disorders.

Mitochondria are pivotal in the biochemical initiation of apoptosis by the release of cytochrome $c$ (Cyt-C) into the cytoplasm that in turn activates the caspase cascade and finally leads to apoptosis. The release of Cyt-C may occur secondary to the onset of the mitochondrial permeability transition pore (mPTP) that leads to disruption of the mitochondrial membranes and the release of pro-apoptotic factors. ${ }^{6}$ Although the role of MPTP in controlling downstream apoptotic events such as Cyt- $C$ release is relatively well characterized, the mechanism by which upstream apoptotic signals are transduced to mitochondria with $\mathrm{MPTP}$ as the target remains elusive. It has been shown that movement of proteins in or out of the mitochondria plays an essential role in the regulation of apoptosis. A particular example is the $\mathrm{Bcl}-2$ family proteins that translocate to mitochondria and initiate the release of Cyt-C by controlling mitochondrial membrane integrity. ${ }^{7}$ Other identified proteins that target to mitochondria through translocation with negative or positive regulatory effects on apoptosis include the transcription factors, ${ }^{8}$ protein kinases, ${ }^{9}$ gap junction proteins $^{10}$ as well as the cytoskeleton-related proteins. ${ }^{11}$ Activation of these proteins in apoptosis can be achieved by post-transcriptional modifications such as phosphorylation or dephosphorylation. ${ }^{8-12}$ It is conceivable that a potential regulatory cross-talk among them might exist to maintain the balance between cell death and survival.

Microtubule-associated proteins (MAPs) are primarily recognized as cytosolic proteins that bind to tubulin and stimulate their polymerization. The assembly-promoting

\footnotetext{
${ }^{1}$ Endocrinology Department, Southwest Hospital, State Key Laboratory of Trauma, Burns and Combined Injury, Third Military Medical University, Chongqing, China; ${ }^{2}$ Institute of Burn Research, Southwest Hospital, State Key Laboratory of Trauma, Burns and Combined Injury, Third Military Medical University, Chongqing, China; ${ }^{3}$ Department of Gynecology and Obstetrics, Daping Hospital, Third Military Medical University, Chongqing, China; ${ }^{4}$ Center for Cardiovascular Research and Alternative Medicine, University of Wyoming College of Health Sciences, Laramie, WY, USA and ${ }^{5}$ Epithelial Cell Biology Research Center, Key Laboratory for Regenerative Medicine of Ministry of Education of China (Jinan University-Chinese University of Hong Kong), School of Biomedical Sciences, The Chinese University of Hong Kong, Shatin, Hong Kong

${ }^{*}$ Corresponding author: J Zhang or Y Huang, Institute of Burn Research, Southwest Hospital, State Key Laboratory of Trauma, Burns and Combined Injury, Third Military Medical University, Chongqing 400038, China. Tel/Fax: +86 236546 1696; E-mail: japzhang@ aliyun.com or yshuang.tmmu@gmail.com ${ }^{6}$ These authors contributed equally to this work.

Abbreviations: Cyt-C, cytochrome $C$; MPTP, mitochondrial permeability transition pore; MAP, microtubule-associated protein; MBD, microtubule binding domain; p-MAP4, phosphor-MAP4; BSA, bovine serum albumin; Glu, glutamate; Ala, alanine; CsA, cyclosporin A; CM, cardiomyocyte; MOI, multiplicity of infection; IOD, integrated optical density; ANOVA, one-way analysis of variance

Received 20.4.14; revised 09.7.14; accepted 23.7.14; Edited by C Munoz-Pinedo
} 
MAPs include tau, MAP2 and MAP4, and MAP4 is ubiquitously expressed in nonneural cells with an important role in microtubule dynamics, as indicated in our and other previous studies. ${ }^{13-16}$ Recent reports raise the possibility that MAPs may be involved in the regulation of mitochondrial function or apoptosis. Purified mitochondria from rat brain were found to contain MAP1 and MAP2. ${ }^{17}$ MAP1 light chain 3 mediates caspase- 8 activation and apoptosis induced by protein degradation inhibition ${ }^{18}$ or cigarette smoke-induced apoptosis and emphysema. ${ }^{19}$ Tau accumulating on mitochondria could have direct repercussions on mitochondrial function in various aspects of the pathogenesis of Alzheimer's disease. ${ }^{20-22}$ However, the definite function(s) and mechanism of MAPs with respect to mitochondria or apoptosis have not been clarified yet.

MAP-microtubule binding is regulated through MAP phosphorylation, leading to the detachment of MAP from microtubules and destabilization of the microtubule as a consequence. ${ }^{13-15}$ The phosphorylation sites of human MAP4 at S696, S768 and S787 (the corresponding sites in rats are predicted to be $S 668, S 738$ and S761) in the prolinerich region of microtubule-binding domain (MBD) have been suggested as the critical sites determining its detachment from microtubules. ${ }^{16,23,24}$ In our previous study, we have shown MKK6/p38 MAPK as the upstream mechanisms responsible for hypoxia-induced MAP4 phosphorylation with subsequent microtubule depolymerization in cardiomyocytes. ${ }^{16}$ Although much attention has been paid to the functional details of MAP4 on microtubules in the past decades, no question has been asked about the destiny of MAP4 itself after it is phosphorylated and detached from microtubules.

In the present study, we find unexpectedly that hypoxia robustly induces phosphorylation and translocation of the cytosolic MAP4 to mitochondria. Translocated phosphorMAP4 (p-MAP4) leads to mPTP opening, mitochondrial dysfunction and apoptosis.

\section{Results}

Identification and localization of MAP4 in mitochondria. We have previously detected MAP4 phosphorylation with microtubule depolymerization as a consequence in hypoxic cardiomyocytes. ${ }^{16}$ In close examination of the intracellular distribution of MAP4 after treatment with hypoxia, we found unexpectedly a time-dependent increase in MAP4 signal overlapping with a mitochondria tracker, MitoTracker Red CM-H2XROS (Figures $1 \mathrm{a}$ and $\mathrm{b}$ ), whereas a timedependent decrease in this signal overlapping with tubulin was observed expectedly (Figures 1c and d). Mitochondrial translocation of MAP4 was further confirmed by analyzing the expression of MAP4 in subcellular fractions under normoxia condition by western blots. E-cadherin, VDAC, calnexin, histone and GAPDH were used as the marker proteins for plasma membrane, mitochondria, endoplasmic reticulum, nucleus and cytosol, respectively. The results showed that MAP4 was indeed found in mitochondrial fraction (Figure 1e). These results demonstrated for the first time the expression of MAP4 in neonatal cardiomyocyte mitochondria.
Prompt translocation of MAP4 to mitochondria upon its phosphorylation under hypoxia stress. We and others have determined that the amino acid residues S696, S768 and S787 in MAP4 are the critical sites responsible for MAP4 binding to tubulin; phosphorylation of these sites leads to MAP4 dissociation from tubulin. ${ }^{16,23-24}$ To see whether the above observed overlapping of MAP4 signal with mitochondria (Figures 1a and b) was a reflection of the MAP4 shift from cytoplasm to mitochondria upon its phosphorylation and dissociation from tubulin, MAP4 phosphorylation and its subcellular distribution were investigated by western blots in cultured cardiomyocytes with or without hypoxia treatment. As shown in Figure 2a, MAP4 showed weak basal levels of phosphorylation at S696, S768 and S787 in cultured neonatal cardiomyocytes under normoxia condition. However, hypoxia $\left(\begin{array}{ll}1 \% & \mathrm{O}_{2}\end{array}\right)$ induced a robust phosphorylation at all the three residues in a time-dependent manner, with the total MAP4 levels unchanged. Analysis of the subcellular distribution of MAP4 showed that although both total MAP4 and p-MAP4 in the mitochondria were at a low level under normoxia condition, they were all dramatically increased after hypoxia treatment, concomitant with a striking decrease of total MAP4 and some decrease of $\mathrm{p}-\mathrm{MAP} 4$ in the remaining fractions (Figure $2 b$ ). In Figures $2 a$ and b, rabbit polyclonal antibody against p-MAP4 (S696) or p-MAP4 (S787) was raised by ourselves using the C-terminal 14 amino acids (PNKEPPP(pS)PEKKAK or KVAEKRT(pS)PSKPSSA and the respective nonphosphorylated peptides) conjugated to bovine serum albumin (BSA). The preparation and verification of antibodies are shown in Figure 2c. These data, thus, revealed an accumulation of p-MAP4 in mitochondria under hypoxia stress, likely because of the prompt translocation of MAP4 from the cytosol to mitochondria upon being phosphorylated.

To confirm whether the phosphorylation status of MAP4 indeed influences its ability to translocate to mitochondria, two MAP4 mutants were generated that mimicked either the phosphorylated or nonphosphorylated forms by changing S696, S768 and S787 to glutamate (MAP4(Glu)) ${ }^{16}$ or alanine (MAP4(Ala)), respectively. Mutant forms of MAP4 (Glu and Ala) or wild-type MAP4 (MAP4-GFP) were expressed at comparable levels in neonatal cardiomyocytes (Figure 2d) and examined by confocal microscopy (Figure 2e). Interestingly, the overlap of transfected MAP4 with mitochondria was dramatically increased in MAP4(Glu), but decreased in MAP4(Ala) transfectants, as compared with cells transfected with wild-type MAP4 (MAP4-GFP) (Figures $2 e$ and $f$ ). This differential localization of MAP4(Glu) and MAP4(Ala) was further confirmed by western blots on subcellular fractions (Figure $2 \mathrm{~g}$ ), showing that MAP4(Glu) primarily concentrated in mitochondria, whereas MAP4(Ala) preferably localized in cytoplasm. These data, collectively, confirmed that the phosphorylation of MAP4 at S696, S768 and S787 promoted the translocation of MAP4 to mitochondria.

Mitochondrial translocation of p-MAP4 is an early step required for apoptosis in hypoxic cardiomyocyte. Protein movement into mitochondria has been reported to be either a cellular adaptive or disruptive response to stimuli. ${ }^{8-12}$ 
a
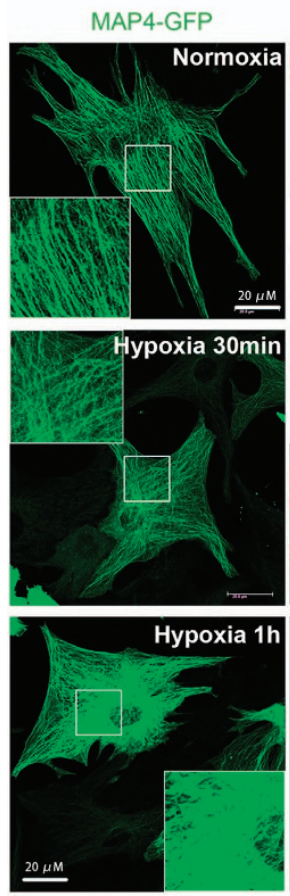

b

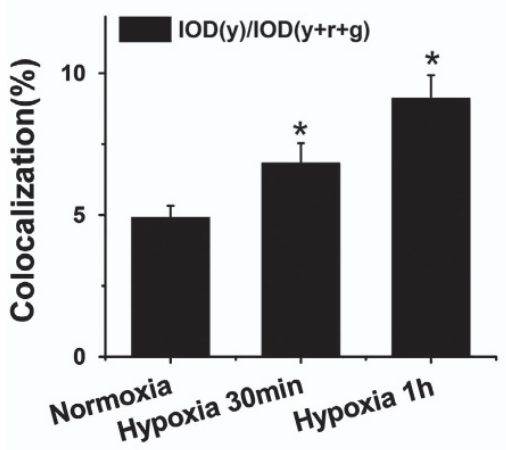

MitoTracker Red
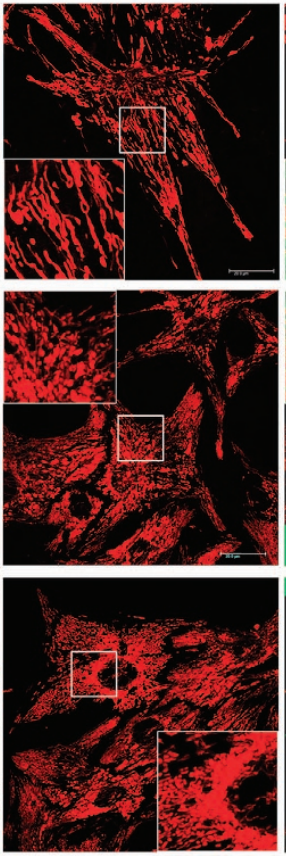

Merged
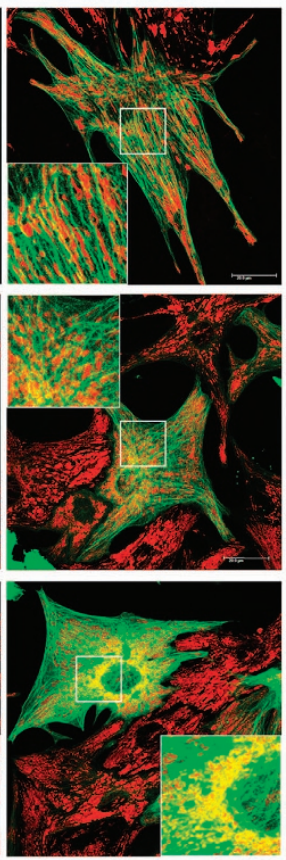

C
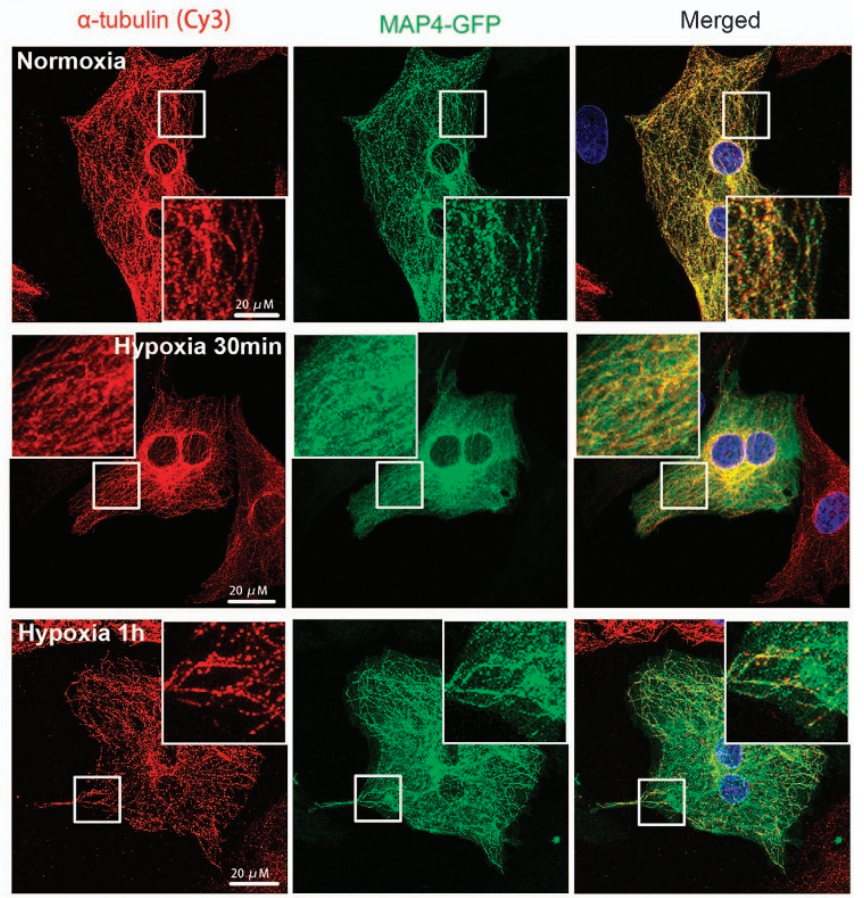

Figure 1 Identification and localization of MAP4 in mitochondria. (a and $\mathbf{b})$ Representative confocal immunofluorescence images (a) or quantitative analysis (b) showing increased colocalization of MAP4 with mitochondria under hypoxia stress. (c and d) Representative confocal immunofluorescence images (c) or quantitative analysis (d) showing decreased colocalization of MAP4 with microtubule under hypoxia stress. Yellow color reflects the overlap of MAP4-GFP (green) with mitochondrial tracker (red) or microtubule (red). Scale bar, $20 \mu \mathrm{m}$. For quantitative analysis, 10 cells were selected and calculated at random $(n=3)$. Representative immunoblots showing subcellular location of MAP4 with corresponding markers (e). Mem, plasma membrane; Mito, mitochondria; ER, endoplasmic reticulum; Nuc, nucleus; Cyto, cytosol; Pero, peroxysome; TME, total mitochondrial extracts; OM, outer membrane; IMS, intermembrane space fractions; IM, inner membrane; MA, matrix fractions. All data are means \pm S.E.M.; ${ }^{*} P<0.01$ versus normoxia control by ANOVA with post hoc analysis

To explore the biological function of hypoxia-induced mitochondrial translocation of p-MAP4, we first examined the effect of mutant MAP4(Glu) on cellular viability. Neonatal cardiomyocytes overexpresssing MAP4(Glu) showed reduced cell viability under either normoxia or hypoxia condition in a time-dependent manner, indicating that p-MAP4 is not in favor of cell survival (data not shown). We then examined whether the p-MAP4-mediated reduction in cell viability resulted from apoptosis or necrosis. By double staining of living cells with annexin V-Cy3 (Red) and SYTOX dye (Green), we could distinguish early apoptosis (annexin $\mathrm{V}+$ / SYTOX - ), late apoptosis (annexin $\mathrm{V}+/$ SYTOX + ) or necrosis (annexin V-/ SYTOX + ) in neonatal cardiomyocytes under normoxia condition or after hypoxia $\left(1 \% \mathrm{O}_{2}\right)$ treatment (Figure 3a). Apoptosis (annexin $\mathrm{V}+$ ) was induced by $3 \mathrm{~h}$ of hypoxia treatment in control cells (CMV-null) that could be significantly increased by MAP4(Glu) under both normoxia and hypoxia conditions (Figures $3 a$ and b). MAP4(Ala) overexpression largely abolished the hypoxiainduced increase in annexin $\mathrm{V}+$ cells (Figures $3 \mathrm{a}$ and $\mathrm{b}$ ). Interestingly, neither MAP4(Glu) nor MAP4(Ala) had obvious effect on hypoxia-induced necrosis (annexinV - /SYTOX +) (Figure 3c), suggesting the involvement of p-MAP4 in hypoxia-induced apoptosis but not necrotic cell death. 
a

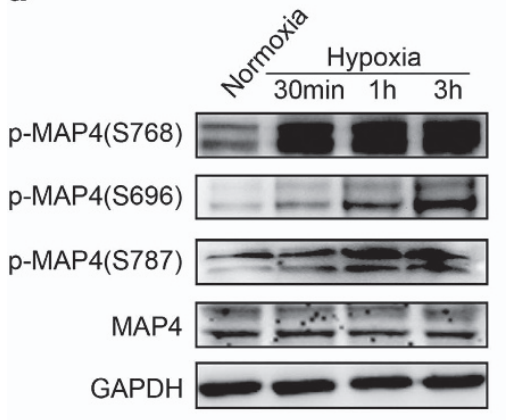

C

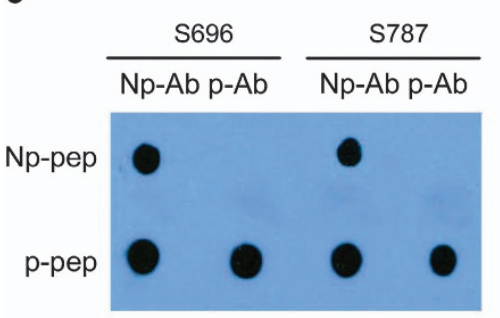

Dot blot b

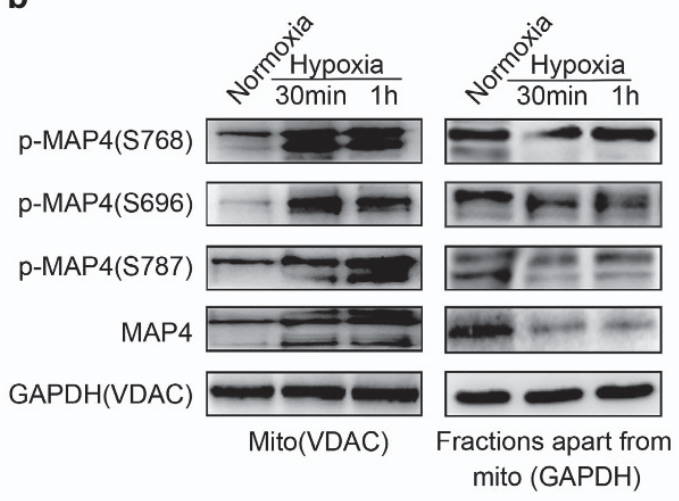

d

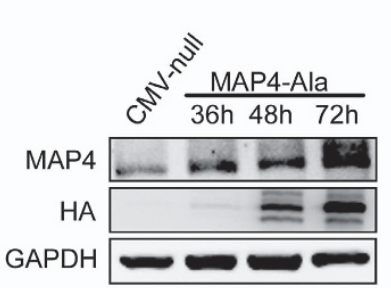

e

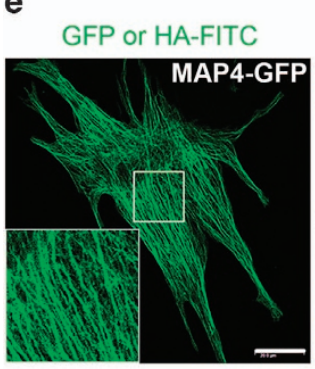

MitoTracker Red

Merged
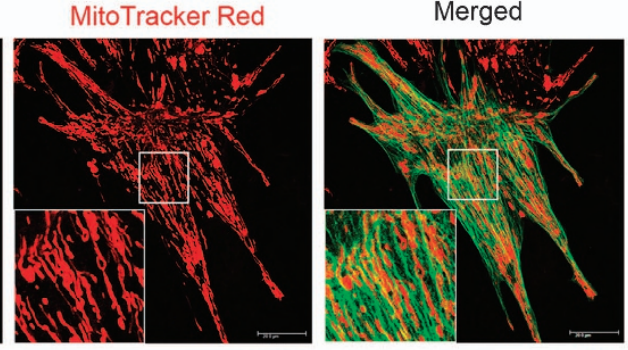

MAP4(Glu)-HA
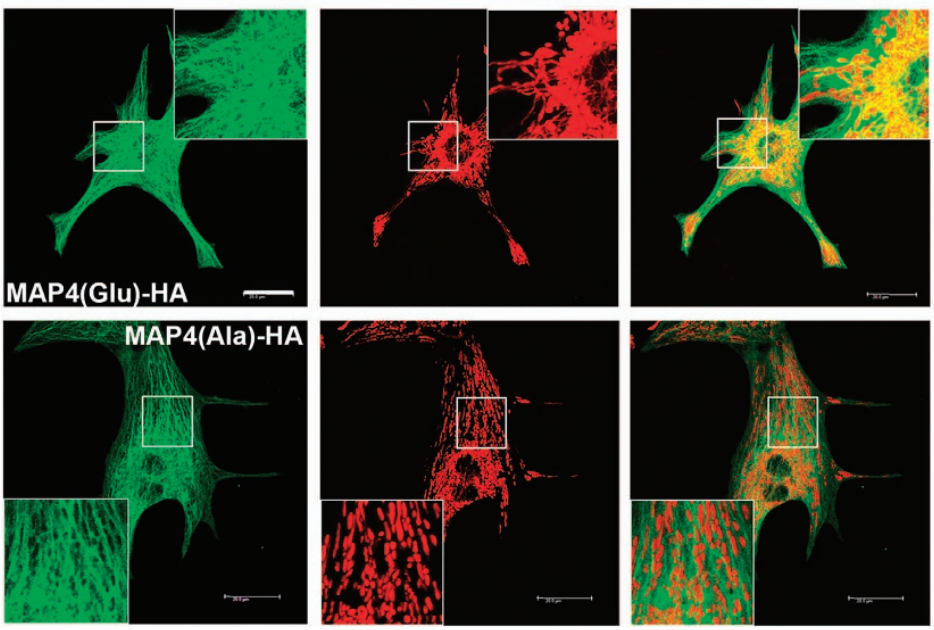

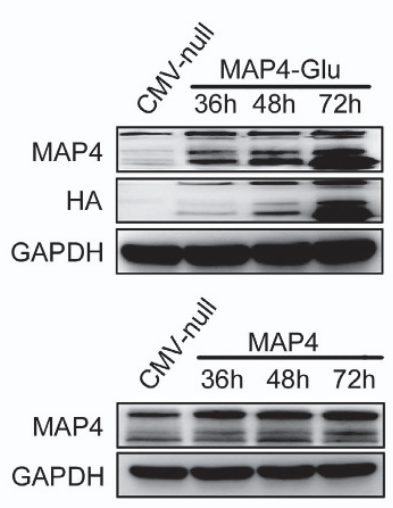

f

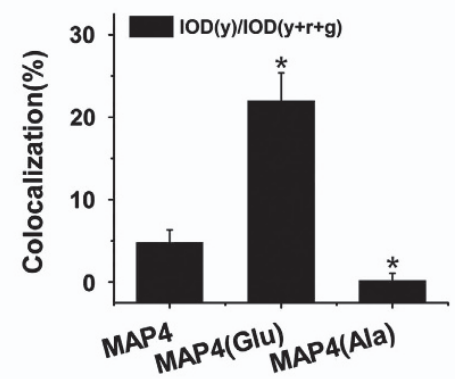

g

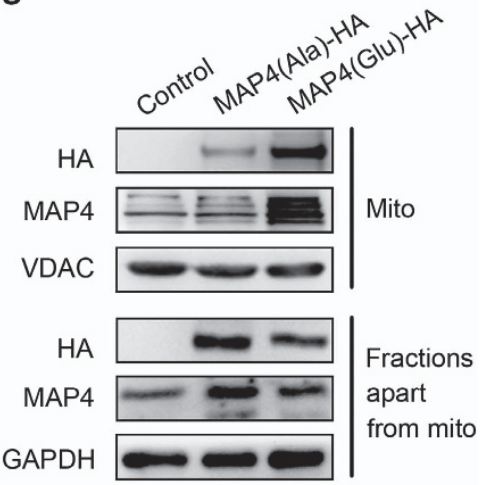


This notion was further supported by TUNEL staining experiments (Figures $3 d$ and e) showing the strongest apoptotic activities in MAP4(Glu) cells but minimal apoptotic levels in MAP4(Ala) cells under normoxia or hypoxia conditions. These findings identify p-MAP4 as a modulator of apoptosis, but not necrosis, in the hypoxic neonatal cardiomyocytes.

Effect of p-MAP4 translocation on apoptotic mitochondrial changes in cardiomyocytes. Mitochondria are pivotal in the biochemical execution of apoptosis. Not only do they provide the energy essential for the completion of apoptosis, they also release important pro-apoptotic factors into the cytosol. Mitochondria are the primary site of the p-MAP4, suggesting its key role in the action of pro-apoptosis regulation. Increased release of Cyt-C, an important pro-apoptotic factor, from mitochondria (Figure 4A) and activation of caspase-9 (Figure 4B) were observed in MAP4(Glu) but reduced in MAP4(Ala) cells, indicating that the mitochondrial pathway may be responsible for p-MAP4mediated myocyte apoptosis.

The release of Cyt-C may occur secondary to the onset of the MPTP that leads to mitochondrial dysfunction with the loss of $\Delta \Psi \mathrm{m}$ and ATP, and the ultimate cell death. ${ }^{6}$ We asked whether hypoxia-induced mitochondrial translocation of p-MAP4 plays a role in these events in neonatal cardiomyocytes. We assessed mPTP status by the calcein-AM loading/ $\mathrm{CoCl}_{2}$ quenching technique, where the decrease in mitochondrial calcein signals was a correlate of MPTP opening. Indeed,
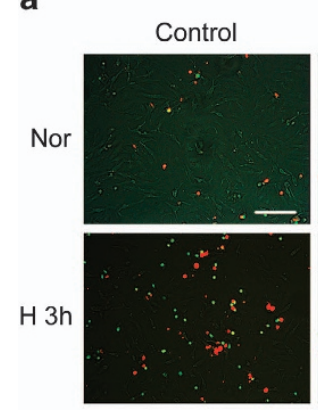

d
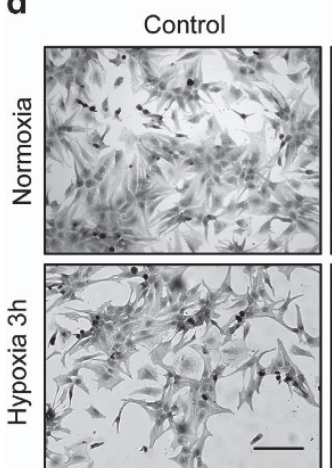

\begin{abstract}
$\operatorname{MAP} 4(\mathrm{Glu})$
\end{abstract}

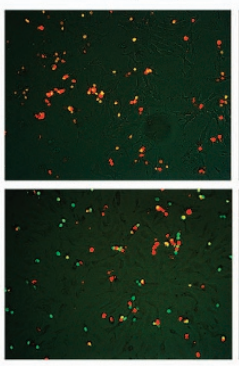

MAP4(Glu)

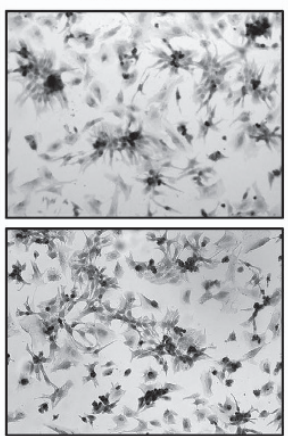

b

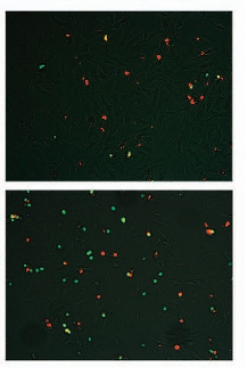

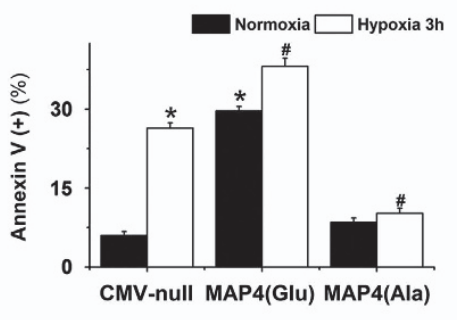

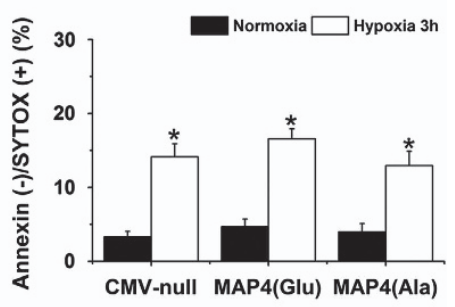

e
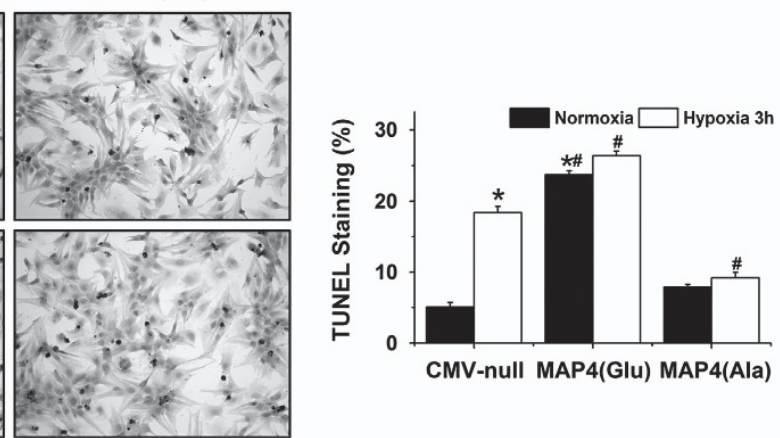

Figure 3 Mitochondrial translocation of p-MAP4 is an early step required for apoptosis in hypoxic cardiomyocyte. (a) Apoptotic or necrotic cardiomyocytes death detected by annexin V-Cy3/ SYTOX green dye staining. Apoptotic cells show red (early apoptosis) or yellow (late apoptosis); necrotic cells are green. Bar, $100 \mu \mathrm{m}$. (b and $\mathbf{c}$ ) Data summary of apoptotic (b) or necrotic (c) cells detected by Annexin V-Cy3/ SYTOX green dye staining. (d and e) Cardiomyocytes apoptosis assessed by TUNEL staining. Representative images showing apoptosis by TUNEL staining (d) and quantitative analysis (e) in normoxic or hypoxic cardiomyocytes with or without overexpression of MAP4(Glu) or MAP4(Ala). Bar, $100 \mu$ m. ${ }^{*} P<0.01$ versus normoxia CMV-null; ${ }^{\#} P<0.01$ versus hypoxia CMV-null

Figure 2 Prompt translocation of MAP4 to mitochondria upon its phosphorylation under hypoxia stress. (a) Hypoxia-induced phosphorylation of MAP4 at S696, S768 and S787 in cultured cardiomyocytes, with no change in total MAP4. (b) Both total MAP4 and p-MAP4 dramatically increased in mitochondria in cultured cardiomyocytes after hypoxia treatment, concomitant with a striking decrease of the total MAP4 and some decrease of the p-MAP4 in the remaining fractions. (c) The preparation and verification of anti-p-MAP4(S696) and anti-p-MAP4(S787) polyclonal antibody. Np-pep, non-phos-peptide; p-pep, phos-peptide; Np-Ab, non-phos-antibody; p-Ab, phos-antibody. (d) Confirmation of adenovirus transfection at comparable levels in cardiomyocytes. Total cell extracts from cardiomyocytes after transfecting MAP4, MAP4(Glu) or MAP4(Ala) adenovirus were analyzed by western blotting. (e and f) Representative confocal immunofluorescence staining (e) and quantitative analysis (f) showing that colocalization of MAP4 with mitochondria was increased by MAP4(Glu), but decreased by MAP4(Ala) mutant, as compared with wild-type controls (MAP4-GFP). Cardiomyocytes were double labeled for mitochondria (MitoTracker Red CM-H2XRos) and mutant MAP4 (Cy3 conjugated anti-HA antibody) or MAP4-GFP. Bars, $20 \mu \mathrm{m}$. For quantitative analysis, 10 cells were selected and calculated at random; $n=3$ performed in triplicate. (g) Differential localization of MAP4 mutants confirmed by western blots. MAP4(Glu): MAP4(S696G, S678G, S787G), MAP4(Ala): MAP4(S696A, S678A, S787A). All data are means \pm S.E.M.; ${ }^{*} P<0.01$ versus MAP4 control 
A

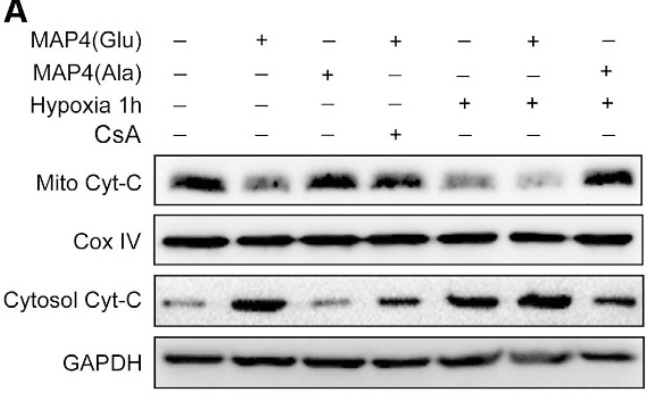

B

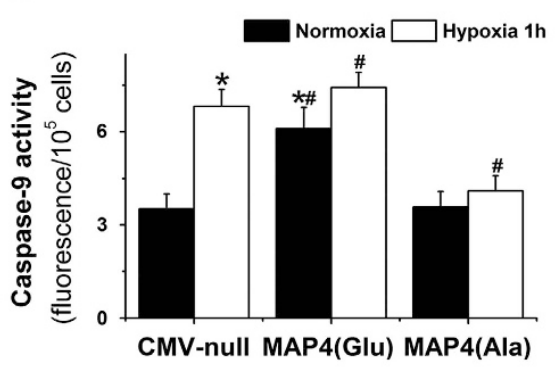

C

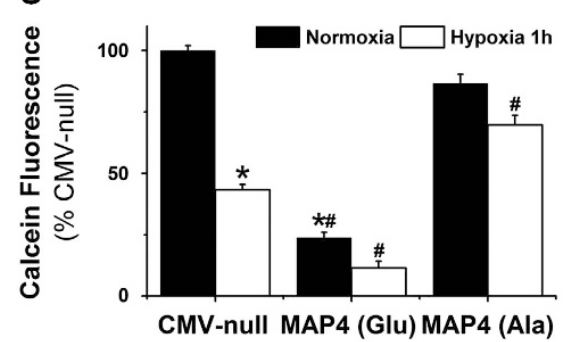

$\mathbf{F}$

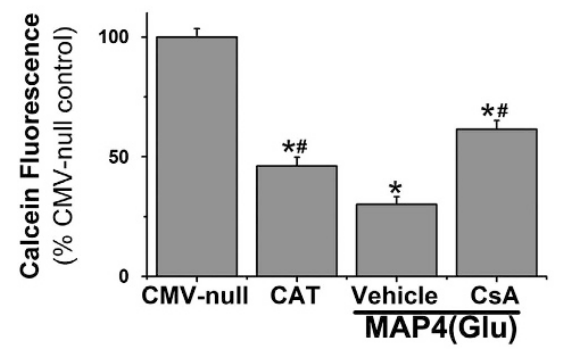

D

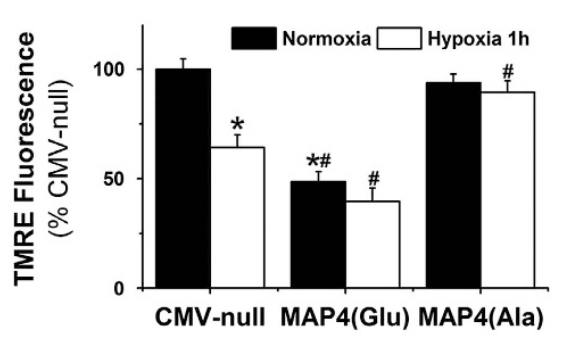

E

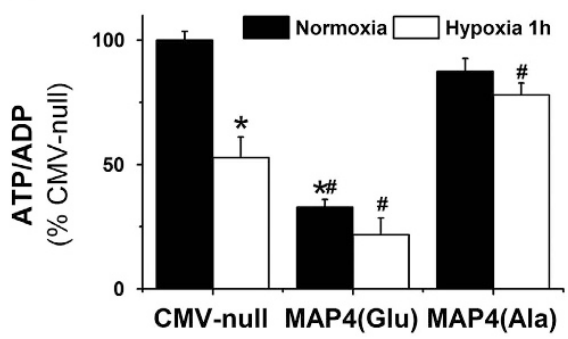

G
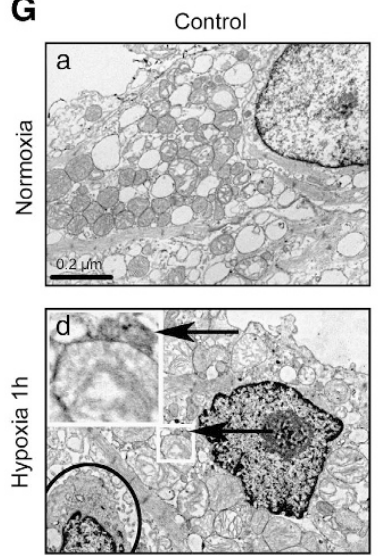
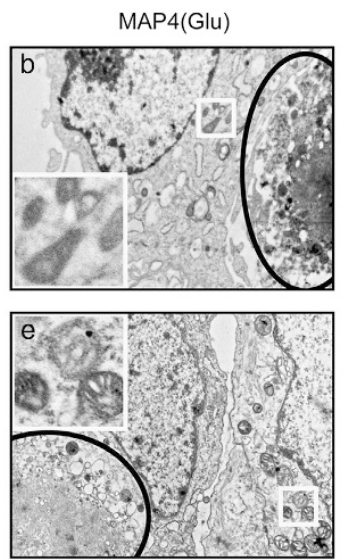
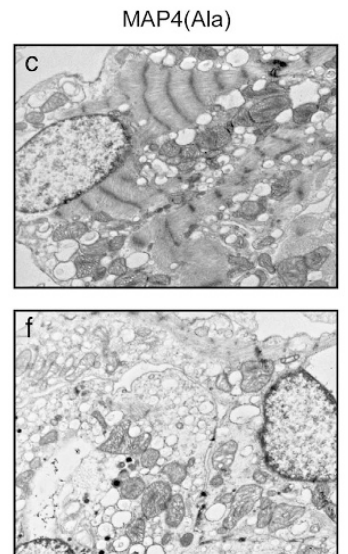

Figure 4 Effect of p-MAP4 translocation on apoptotic mitochondrial changes in cardiomyocytes. (A) Detection of mitochondrial Cyt-C release by western blots. (B) Caspase-9 activity detected by Caspase-9 Fluorometric Assay Kit. (C and D) Assay of mPTP opening by calcein fluorescence (C) or mitochondrial membrane potential by tetramethylrhodamine ethyl ester (TMRE) (D). ${ }^{*} P<0.01$ versus normoxia CMV-null; ${ }^{\#} P<0.01$ versus hypoxia CMV-null. (E) ATP/ADP detected by ADP/ATP ratio assay kit. (F) mPTP opening, as reflected by the decrease of calcein fluorescence, was induced by MAP4(Glu) that could be inhibited by CsA (5 $\mu$ M). (G) Representative electron microscope images showing typical apoptosis (black circled area in b, $\mathrm{d}$ and e) and swelling (enlarged white square area in d) or shrinked (black arrow in $\mathrm{d}$ and enlarged white square area in $b$ and e) mitochondria induced by hypoxia or MAP4(Glu). a is the normal control, and the MAP4(Ala) protects the cells from the apoptosis (c, $f$ ). ${ }^{*} P<0.01$ versus CMV-null control; ${ }^{~} P<0.01$ versus MAP4(Glu) overexpression vehicle group. MAP4(Glu): MAP4(S696G, S678G, S787G), MAP4(Ala): MAP4(S696A, S678A, S787A). A total of 20 images are selected at random and analyzed for each group. All data are mean \pm S.E.M. of six independent experiments performed in triplicate

the hypoxia-induced decrease in mitochondrial calcein signals was significantly aggravated by the phosphorylation mutant MAP4(Glu), but almost completely reversed by the nonphosphorylation mutant MAP4(Ala), indicating that MPTP opening could be controlled by the phosphorylation status of MAP4 (Figure 4C). We corroborated our findings by a similar assay where a decrease in fluorescence corresponds to a loss of $\Delta \Psi \mathrm{m}$ and the ratio of ATP/ADP. Neonatal cardiomyocytes overexpresssing MAP4(Glu) were significantly more sensitive to $\Delta \Psi \mathrm{m}$ and ATP loss in response to hypoxia challenge compared with vector control, whereas overexpresssion of MAP4(Ala) rendered the cells more resistant to such $\triangle \Psi \mathrm{m}$ or ATP loss (Figures 4D and E). It is noteworthy that MAP4(Glu) could mimic hypoxic condition inducing both MPTP opening and $\Delta \Psi \mathrm{m}$ or ATP loss in normoxia-cultured cells (Figures $4 \mathrm{C}-\mathrm{E}$ ), further supporting a role of p-MAP4 in hypoxia-induced mitochondrial dysfunction. To test whether the observed apoptosis above was attributed to MPTP-related mitochondrial dysfunction, cells were treated with cyclosporin A (CsA), a known mPTP inhibitor. The results showed that CsA ( $5 \mu \mathrm{mol} / \mathrm{l})$ significantly inhibited MAP4(Glu)-induced calcein leakage (Figure $4 \mathrm{~F}$ ) and $\mathrm{Cyt}-\mathrm{C}$ release from mitochondria (Figure 4A), suggesting the involvement of mPTP mechanism in p-MAP4-mediated neonatal cardiomyocyte apoptosis.

The alteration of mitochondrial morphology and distribution induced by p-MAP4 were further supported by electron microscopic observations (Figure 4G). After hypoxia treatment $\left(1 \% \mathrm{O}_{2}, 1 \mathrm{~h}\right)$, mitochondria showed rarefaction of 
A
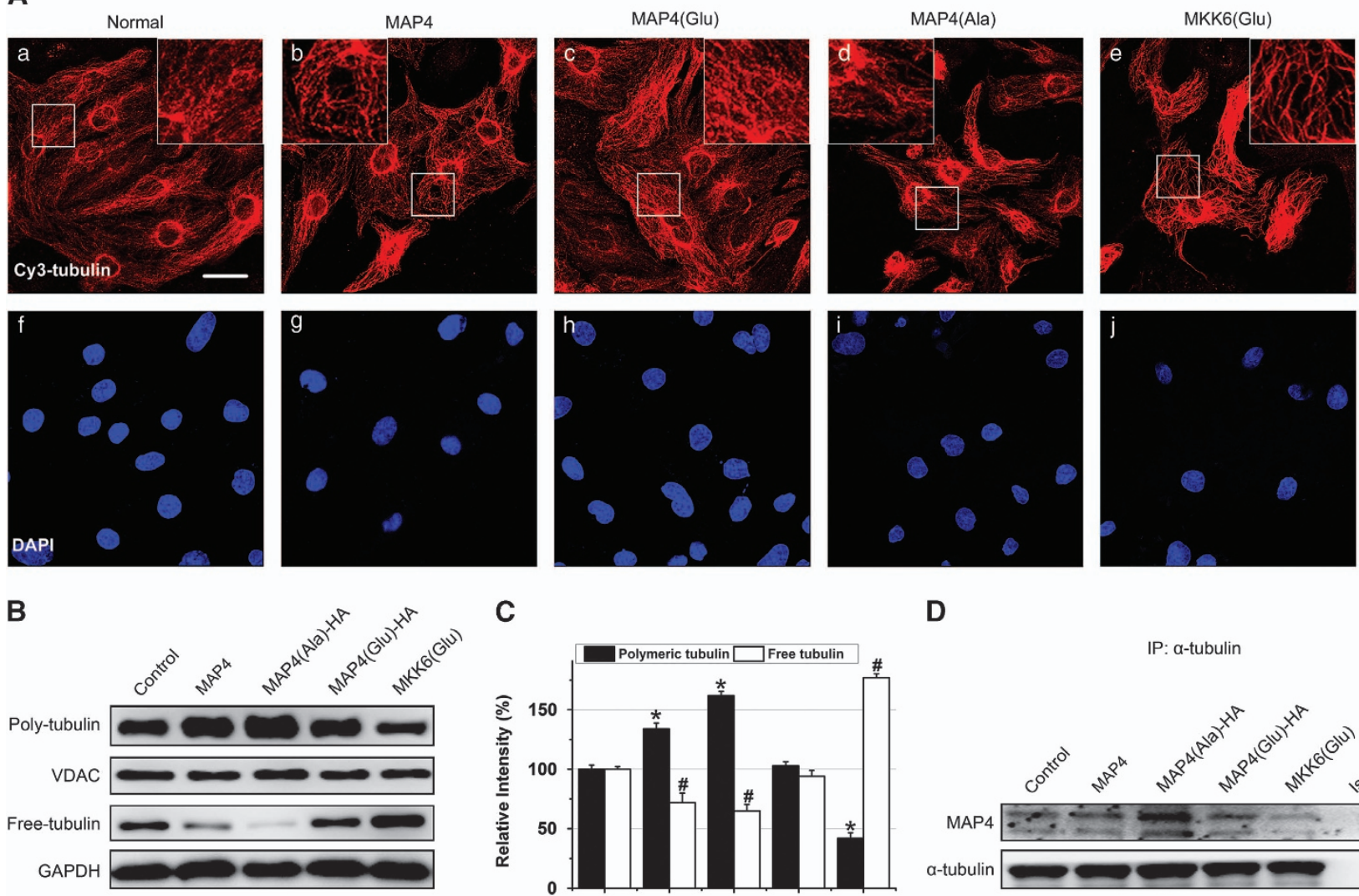

C

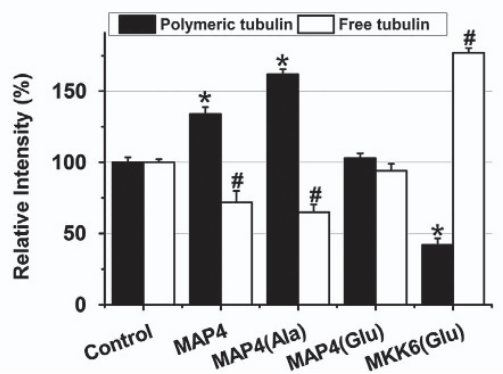

D

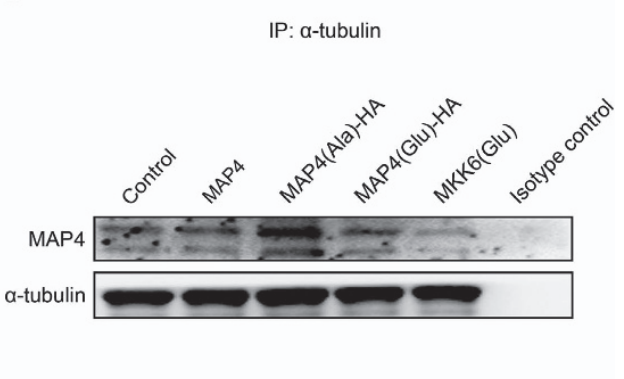

Figure 5 Mitochondrial p-MAP4 leads to apoptosis independently of microtubule assembly. (A) Representative immunostaining results showing more disassembled microtubule networks in cardiomyocytes transfected with $\operatorname{MKK} 6(\mathrm{Glu})(\mathrm{e}, \mathrm{j})$ compared to the normal $(\mathrm{a}, \mathrm{f})$. The boxed areas are shown at higher magnification in the inserts to illustrate details. The wild-type MAP4 (b, g) or MAP4(Ala) (d, i) lead to condensed microtubules, while the MAP4(Glu) $(c, h)$ has no effect on the microtubule structure. Bars, $25 \mu \mathrm{m}$. (B and $\mathbf{C}$ ) Representative blotting results (B) and quantitative analysis (C) showing that polymeric or free tubulin was not affected by MAP4(Glu), but changed by wildtype MAP4, MAP4(Ala) or MKK6(Glu) in cardiomyocytes. Herein, VDAC in mitochondrial fractions was chosen as the control for polymerized tubulin, and GAPDH in the cytosolic fractions was chosen as the internal control for free tubulin. (D) Determination of MAP4 binding to tubulin in cells with wild-type MAP4, MAP4(Glu), MAP4(Ala) or MKK6(Glu) overexpression by IP. Data are mean \pm S.E.M. of six independent experiments performed in triplicate; ${ }^{*} P<0.01$ versus polymeric tubulin control; ${ }^{\#} P<0.01$ versus free tubulin control by ANOVA post hoc tests

mitochondrial matrix and ruptures of internal cristae (white boxed area), whereas some others showed condensation and increase of electron density (black arrow) (Figure 4Gd). Mitochondria responded to MAP4(Ala) by readopting an orthodox configuration under hypoxic conditions (Figure 4Gf). MAP4(Glu) induced progressively degenerative mitochondria with condensation and collapse of internal cristae (Figures $3 \mathrm{~Gb}$ and e). Furthermore, the classic apoptotic morphological changes of cardiomyocytes could be seen in hypoxic or MAP4(Glu) groups by electron microscopic observations, which were also consistent with the annexin $\mathrm{V}$ and TUNEL staining in Figure 3.

Mitochondrial p-MAP4 leads to apoptosis independently of microtubule assembly. The phosphorylation and mitochondrial translocation of p-MAP4 follows its detachment from microtubules that may result in microtubule instability. In order to exclude the effect of microtubule depolymerization on the mitochondria homeostasis induced by MAP4 phosphorylation, we observed microtubule changes after transfecting the MAP4 or mutant MAP4. MKK6(Glu), the persistently activated form of MKK6 that activated the downstream effector p38/MAPK and induced MAP4 phosphorylation with subsequent microtubule depolymerization in cardiomyocytes, ${ }^{16}$ was applied as the positive control. Neonatal cardiomyocytes subjected to MAP4 or MAP4(Ala) showed clear signs of curl and buckle in the vicinity of the nuclei and along the edges of the cell that was attributed to microtubule hyperpolymerization (Figures $5 \mathrm{Ab}$ and $\mathrm{d}$ ). MKK6(Glu) resulted in very significant microtubule depolymerization represented by a thin and disrupted residual network (Figure 5Ae), whereas MAP4(Glu) had no significant effect on the microtubules (Figure 5Ac). The tubulin fractions quantification confirmed that polymeric tubulin levels significantly decreased in the MKK6(Glu) group, but increased in both MAP4 and MAP4(Ala) groups on the contrary. In the MAP4(Glu) group, the polymeric tubulin levels had no significant changes (Figures $5 \mathrm{~B}$ and $\mathrm{C}$ ). The interaction between mutant MAP4 and tubulin increased in MAP4 or MAP4(Ala) overexpression group, whereas it decreased in 
the MKK6(Glu) group, in which MAP4 were hyperphosphorylated. MAP4(Glu) overexpression had no effect on the interaction because of its very slight binding with tubulin indicated by the HA-tag (Figure 5D). This observation suggests that p-MAP4 can lead to apoptosis through its mitochondrial translocation independently of microtubule assembly. It may be because MAP4(Glu) has little binding ability to tubulin and thus could not change the microtubule dynamics. ${ }^{13-15}$

\section{Discussion}

MAP4, the cytosolic microtubule-binding protein, is previously recognized as the key regulator in microtubule dynamics involving in a wide range of physiological and cellular functions such as intracellular transport, axonal growth, cell migration and mitosis. ${ }^{25-27}$ We have recently addressed the importance of the MAP4-regulated microtubule stability in energy supply of neonatal cardiomyocytes under hypoxia condition, ${ }^{28,29}$ and further indicated MKK6/p38 MAPK as the upstream signal responsible for hypoxia-induced MAP4 phosphorylation leading to microtubule instability. ${ }^{16}$ Here we present our unexpected finding that cytosolic MAP4, upon phosphorylation, translocates to mitochondria in neonatal cardiomyocytes as responded to hypoxia. Further study shows that hypoxia-induced mitochondrial translocation of MAP4 results in mitochondrial dysfunction and cardiomyocyte apoptosis. In all, our study identifies MAP4 as a novel mediator of neonatal cardiomyocyte apoptosis.

Although it has long been recognized that phosphorylation of MAPs results in their detachment from the microtubules, usually as a response to stress such as ischemia and hypoxia, ${ }^{16,30}$ their destiny after detachment remains unknown. The present study reveals for the first time that upon hypoxia-induced phosphorylation, p-MAP4 is not just detached from microtubules, but translocated to mitochondria, eliciting a series of mitochondrial events leading to apoptosis. Another important result of this study is the finding that phosphorylation of MAP4 is a prerequisite for its translocation to mitochondria and the induced mitochondrial-dependent apoptosis in hypoxic cardiomyocytes. Although the exact mechanism underlying mitochondrial translocation of MAP4 requires further investigation, the presence of the basic MAP4 in normoxia mitochondria and the accumulation of p-MAP4 in hypoxic mitochondria are firmly demonstrated in our study by subcellular fractions, confocol microscopy or genetical manipulation experiments. More importantly, although physiologically mitochondrial translocation of p-MAP4 follows its detachment from microtubules, which may result in microtubule instability, we found that mitochondrial dysfunction and apoptosis induced by forced expression of MAP4(Glu) are independent of microtubule disorder (Figure 5), suggesting that phosphorylation of MAP4 alone can result in its mitochondrial translocation leading to apoptosis.

The induction of mPTP opening is a common cause of cardiac mortality under various pathogenic conditions such as hypoxia/ischemia, reperfusion, oxidative stress and cytotoxic drugs. The observed p-MAP4 mitochondrial translocation and the release of Cyt-C from the mitochondria induced by MAP4(Glu) suggests that p-MAP4 may regulate mitochondrial function and apoptosis through its effect on MPTP. The increased $\mathrm{MPTP}$ opening is then proved by the calcein-AM loading/ $\mathrm{CoCl}_{2}$ quenching technique (Figure 4c). Although $\mathrm{mPTP}$ opening is known to be associated with both apoptosis and necrosis, our data suggest that mitochondrial translocation of p-MAP4 only contributes to hypoxia-induced apoptosis (Figures $3 b$ and e), but not necrosis (Figure 3c). Before our study, proteins that translocate into mitochondria with regulatory role in apoptosis through interaction with $\mathrm{mPTP}$ have also been suggested, including the bcl-2 family member $\mathrm{Bax},{ }^{31}$ the inhibitory subunit of NF- $\kappa \mathrm{B},{ }^{32}$ the phospho-GSK-3 $\beta$ and so on. ${ }^{9}$ The mPTP is likely to be a convergence point of the distinct pro- and anti- apoptotic signals, and the detail of mPTP regulation of $\mathrm{p}-\mathrm{MAP} 4$ awaits further investigation. Apart from hypoxia, the phosphorylation and mitochondrial translocation of MAP4 could also be induced by hypoxiareoxygenation treatment (Supplementary Figure $\mathrm{S1}$ ), indicating that MAP4 may represent a general regulatory mechanism underlying both hypoxia and ischemiareperfusion injury in neonatal cardiomyocytes.

In summary, our data provide the first evidence that MAP4 homes to mitochondria in a phosphorylation-dependent manner after detachment from microtubule, and induces or increases intrinsic apoptosis by causing MPTP opening and mitochondrial dysfunction. This novel role has nothing to do with the microtubule dynamics, depicted in Figure 6.

\section{Materials and Methods}

Cardiomyocyte cultures and hypoxia treatment. Neonatal (3-5 days, gender-neutral) Sprague-Dawley rats were applied, and the Institute of Animal Care and Use Committee approved all animal protocols. Neonatal rat ventricular cardiomyocyte (CM) culture and hypoxia treatment were performed as described previously. ${ }^{16}$ Hypoxic conditions were achieved by using an anaerobic jar (Mitsubishi, Tokyo, Japan) and vacuum glove box (Chunlong, Lianyungang, China). Serum-free medium was placed overnight in the vacuum glove box filled with $94 \% \mathrm{~N}_{2}, 5 \% \mathrm{CO}_{2}$ and $1 \% \mathrm{O}_{2}$ and allowed to equilibrate with the hypoxic atmosphere. Cells were placed in the anaerobic jar and subjected to hypoxic conditions by replacing the normoxic medium with the hypoxic medium.

Preparation of mitochondrial and subcellular fractions. Myocardial mitochondria were isolated according to the method described by Jiang et al. ${ }^{33}$ Briefly, rat heart was promptly excised and sequentially placed in ice-cold isolation buffer (mannitol $210 \mathrm{mmol} / /$, sucrose $70 \mathrm{mmo} / /$, Tris $10 \mathrm{mmol} / /$, EDTA $1 \mathrm{mmol} / /$, EGTA $0.5 \mathrm{mmo} / /$; $\mathrm{pH}$ 7.4) with complete protease inhibitor tablets. After being minced and homogenized using $5 \mathrm{ml}$ isolation buffer per $1 \mathrm{~g}$ of tissue, the homogenate was centrifuged at $1000 \mathrm{~g}$ for $10 \mathrm{~min}$, and then the supernatant was collected and again centrifuged at $10000 \mathrm{~g}$ for $10 \mathrm{~min}$ to form a crude mitochondrial pellet. Nycodenz step-gradients (20-34\% w/v; Axis-Shield PoC, Dundee, Scotland) were made by dissolving it in isolation buffer. Crude mitochondrial pellets were suspended in $2.4 \mathrm{ml}$ of $25 \%$ Nycodenz, placed on $1 \mathrm{ml}$ of $34 \%$ and $1.6 \mathrm{ml}$ of $30 \%$ Nycodenz and topped with $1.6 \mathrm{ml}$ of $23 \%$ and $0.6 \mathrm{ml}$ of $20 \%$ Nycodenz. The sealed tubes were centrifuged for $90 \mathrm{~min}$ at $52000 \mathrm{~g}$ at $4^{\circ} \mathrm{C}$. The band at the $23-25 \%$ interface was collected and diluted with the same volume isolation buffer. The mixture was centrifuged at $10000 \mathrm{~g}$ for $10 \mathrm{~min}$, after which the pellet was washed with isolation buffer again and collected as purified mitochondria.

Cell fractionation kit (ab109719; Abcam, Cambridge, MA, USA) was used to isolate the nucleus and the cytosol fractions apart from mitochondria. Plasma Membrane Extraction Kit (K268-50; Biovision, Mountain View, CA, USA) was applied to isolate the membrane protein. The procedure for microsome isolation is as follows: heart tissue was homogenized in double-volume buffer (sucrose $150 \mathrm{mmol} / \mathrm{l}, \mathrm{KCl} 25 \mathrm{mmol} / \mathrm{l}$, Tris- $\mathrm{HCl} 100 \mathrm{mmol} / \mathrm{l}, \mathrm{MgCl}_{2} 5 \mathrm{mmol} / / ; 0^{\circ} \mathrm{C}$, pH 7.4) with complete protease inhibitor cocktail tablets (Roche, Mannheim, Germany) after washing in PBS $\left(4^{\circ} \mathrm{C}\right)$. The homogenate was centrifuged for $10 \mathrm{~min}$ at $15000 \mathrm{~g}$ at $4^{\circ} \mathrm{C}$, and then the supernatant centrifuged at $100000 \mathrm{~g}$ for a further $10 \mathrm{~min}$. The pellet was washed twice in buffer and resuspended in RIPA buffer as the microsome fraction. 


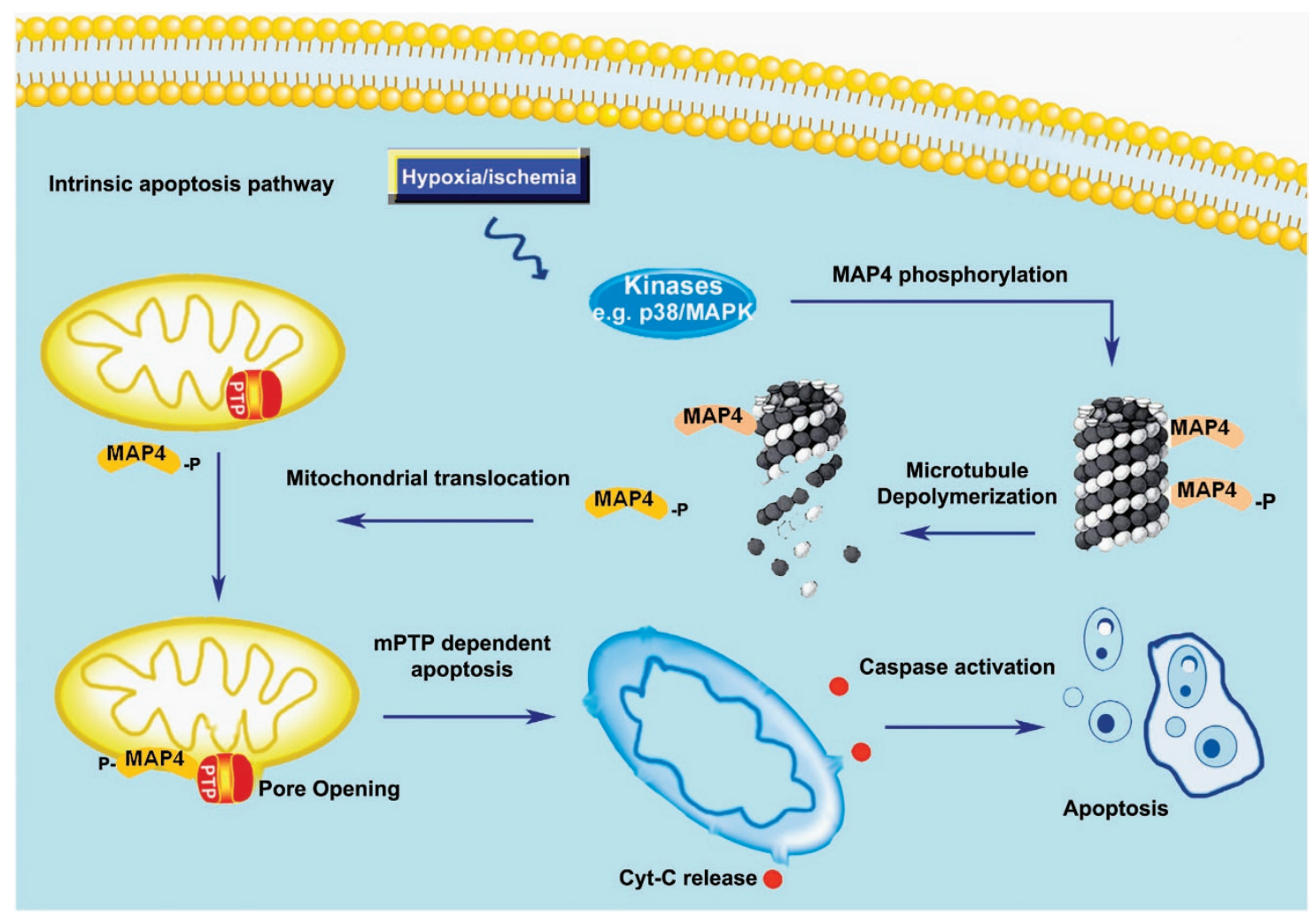

Figure 6 Schematic figures showing p-MAP4 translocation-mediated mitochondrial cardiomyocyte apoptosis under hypoxia stress. MAP4 is primarily bound to cytosolic microtubule and plays an important role in microtubule dynamics. When intrinsic apoptosis pathway is activated by hypoxia, MAP4 dissociates from the tubulin once being phosphorylated, with microtubule instability as a consequence. Phosphor-MAP4 ( $p$-MAP4) is translocated to mitochondria and leads to mPTP opening and apoptosis

Extraction and quantification of tubulin fractions. Polymeric and monomeric tubulin fractions were isolated and quantified using a method previously described. ${ }^{16}$

Immunoblotting analysis. Cardiomyocyte samples were homogenized in RIPA buffer (Sigma-Aldrich, St. Louis, MO, USA) with protease inhibitor tablets. The lysate was centrifuged at $16000 \mathrm{~g}$ at $4^{\circ} \mathrm{C}$ for $15 \mathrm{~min}$ to remove insoluble protein. SDS-PAGE was carried out after equal BCA-based protein loading for each sample using $8-12 \%$ gradient gels. Separated proteins were transferred to PVDF membranes (Millipore, Bedford, MA, USA), blocked with 10\% skimmed milk, and then incubated at $4{ }^{\circ} \mathrm{C}$ overnight with the corresponding primary antibodies and the biotinylated secondary antibody insequence. Specific protein bands were detected using biotinylated horseradish peroxidase in conjunction with an enhanced chemiluminescence detection kit (Amersham Pharmacia, Piscataway, NJ, USA). The following antibodies were used in this experiment: rabbit polyclonal anti-MAP4 (A301-489A, Bethyl Laboratories, Montgomery, TX, USA), p-MAP4(S768) (Ser768, Biolegend, San Diego, CA, USA), Cox IV (ab20393, Abcam), Calnexin (ab22595, Abcam), Catalase (ab1877, Abcam), Histone (Biosynthesis, Lewisville, TX, USA), actin (sc-1616, Santa Cruz Biotechnology, Santa Cruz, CA, USA), GAPDH (KC-5G5, Kangchen, Shanghai, China), mouse monoclonal anti-porin (529534, Calbiochem, San Diego, CA, USA) and Cyt-C (ab13575, Abcam). Rabbit polyclonal antibody against the p-MAP4(S696) or p-MAP4(S787) was raised by ourselves using the C-terminal 14 amino acids (PNKEPPP(pS)PEKKAK or KVAEKRT(pS)PSKPSSA and the respective nonphosphorylated peptides) conjugated to BSA. The preparation and verification of antibodies are shown in Figure 2c.

Site-directed mutagenesis. Primers were designed to generate point mutations of MAP4 S696G, S768G and S787G, or S696A, S768A and S787A through PCR reactions using the QuikChange Multi Site-Directed Mutagenesis Kit (200514, Stratagene, La Jolla, CA, USA; Table 1).
Table 1 Primers for generating mutant microtubule-associated protein 4 (MAP4)

\begin{tabular}{lll}
\hline & \multicolumn{2}{c}{ Primer sequence } \\
\cline { 2 - 3 } $\begin{array}{l}\text { Mutant } \\
\text { sites }\end{array}$ & Reverse $\left(5^{\prime}-\mathbf{3}^{\prime}\right)$ & Forward $\left(\mathbf{5}^{\prime}-\mathbf{3}^{\prime}\right)$ \\
\hline S696G & CTTTTCTGGTTCTGGTGGT & CTACCACCAGAACCAGA \\
& AGCTCCTTGTTTG & AAAGAAAGCAAAG \\
S768G & GGTAGGGTCTCAGGCCTG & GGTAGGGTCTCAGGC \\
& GCAGTAGCAGTG & CTGGCAGTA GCAGTG \\
S787G & TGGATGGCTCGGTCCG & AAGCGGACCGAG CCAT \\
& CTTTTCAGCAAC & CCAAGCCTTCATC \\
S696A & CTTTTCTGGAGCTGGTG & CAAGGAGCTACCACCAG \\
& GTAGCTCCTTG & CTCCAGAAAAG \\
S768A & GGAGCGGTCCGCTTTTCA & GCCTGCTACCCTACCT \\
S787A & GCAACCTTAGTTTCTGTAA & GCAAGAGACCTGAAGC \\
& TTGGCTTTGGCTTCAGGTC & CAAAGCCAATTACAGAA \\
& TCTTGC AGGTAGGGTAGC & ACTAAGGTTGCTGAAA \\
& AGGC & AGCGGA CCGCTCC \\
& &
\end{tabular}

Adenovirus construction. The recombinant adenoviruses were prepared using the AdMax system (Microbix, Toronto, ON, Canada). The CMV-null adenovirus was used as the control. The original and mutated MAP4 CDNAs were then cloned into the pDC315 shuttle adenoviral vector (Genechem, Shanghai, China). The recombinant adenoviruses were prepared using the AdMax system (Microbix) according to the instructions. The multiplicities of infection (MOls) and duration of infection for each adenovirus were determined via immunoblotting to determine the optimum and equal level of MAP4 expression. The CMV-null adenovirus was used as the negative control. In order to determine the effect of cyclosporine (CsA, Sigma-Aldrich) or carboxyatractyloside (CAT, Sigma-Aldrich) 
on the event of MAP4(Glu) overexpression, CsA $(5 \mu \mathrm{M})$ or CAT $(10 \mu \mathrm{M})$ was added into the culture when the GFP of the control transfected cells could be observed.

Electron microscopy. In order to observe the apoptosis and mitochondrial morphology, cardiomyocytes cultured in flasks were scraped and collected by centrifugation at $1200 \mathrm{~g}$ for $10 \mathrm{~min}$. Cardiomyocytes were fixed in $2.5 \%$ glutaraldehyde followed by dehydration, vibratome sliced and recut on a microtome and stained with uranyl acetate and lead citrate overnight. The sections were examined using a transmission electron microscope (TECNAL-12, Philips Electron Optics, Eindhoven, Holland).

Fluorescence and confocal microscopy. For MAP4, tubulin or mitochondrial co-location, cardiomyocytes were transfected with Cellular Lights MAP4-GFP (C10105, Invitrogen, Karlsruhe, Germany) or mutant MAP4, and then incubated with MitoTracker Red CM-H2XRos (100 nM; M7513, Molecular Probes, Eugene, OR, USA) for $30 \mathrm{~min}$ at $37^{\circ} \mathrm{C}$ to show the location of MAP4-GFP and mitochondria. The HA-tagged mutant MAP4-transfected cells were incubated with MitoTracker Red $\mathrm{CM}-\mathrm{H} 2 \mathrm{XR}$ os for $30 \mathrm{~min}$ at $37^{\circ} \mathrm{C}$, followed by fixation with $4 \%$ paraformaldehyde, permeabilized with $0.1 \%$ Triton X-100 in PBS and blocked in $10 \%$ goat serum. The HA-tagged mutant MAP4 was stained with anti-HA monoclonal antibody (H3663, Sigma-Aldrich) and goat anti-mouse secondary antibodies conjugated to cyanine 3 (Cy3; Beyotime, Haimen, China).

To observe the microtubule structure, mouse anti- $\alpha$-tubulin primary antibodies (1:100, SC5286; Santa Cruz Biotechnology) and goat anti-mouse secondary antibodies conjugated to Cy3 $(1: 1000$; Beyotime) were used. The nuclei were then stained for $2 \mathrm{~min}$ with 40,60-diamidino-2-phenylindole (DAPI; $0.5 \mathrm{Ig} / \mathrm{ml}$; SigmaAldrich). For MAP4 and tubulin co-location, the MAP4-GFP-transfected cells were fixed with $4 \%$ paraformaldehyde, permeabilized with $0.1 \%$ Triton X-100 in PBS and blocked in $10 \%$ goat serum. The tubulin was stained as was stated above. Cells were imaged with confocal microscopy (TCS-NT; Leica, St Gallen, Switzerland). The colocalization of MAP4 and mitochondria was expressed as a ratio of the integrated optical density (IOD) of yellow area to the IOD of red + green + yellow by the Image Pro Plus 6.0 software (Media Cybernetics, Silver Spring, MD, USA). For each group, 10 cells were selected and calculated at random, and the experiments were repeated three times, and a mean of 3 means was calculated.

mPTP, $\Delta \Psi \mathrm{m}$, ATPIADP ratio, Cyt-C release and apoptosis assay. MitoProbe Transition Pore Assay Kit (M34153, Molecular Probes), TMRE (Molecular Probes), ADP/ATP ratio assay kit (ab65313, Abcam), Annexin V-Cy3 Apoptosis Detection Kit Plus (K202-100, Biovision), Caspase-9 Fluorometric Assay Kit (K118-100, Biovision) and In Situ Cell Death Detection Kit (11684795910, Roche) were used for $\mathrm{mPTP}, \triangle \Psi \mathrm{m}, \mathrm{ATP} / \mathrm{ADP}$ ratio and apoptosis assay, respectively. A total of 20 images representative of each group were chosen at random, and mean fluorescence intensity was analyzed by Leica Physiology Software (Leica). The experiments were repeated six times in triplicate, and a mean was calculated. For CytC release, mitochondrial and cytosol fractions were separated by mitochondria Isolation Kit (89874, Pierce, Rockford, IL, USA) and the Cyt-C was detected by western blot. The experiment was repeated three times.

Immunoprecipitation. To search for the interaction between MAP4 and tubulin, cells were lysed in RIPA buffer with protease inhibitor tablets. Rabbit anti- $\alpha$-tubulin (SC5286; Santa Cruz Biotechnology) antibodies were incubated with cell lysate for $6 \mathrm{~h}$ at $4^{\circ} \mathrm{C}$, and then the complexes were precipitated with protein $\mathrm{A} /$ G-Sepharose (sc-2003, Santa Cruz Biotechnology) overnight at $4^{\circ} \mathrm{C}$. The precipitates were washed 5 times with PBS at $0^{\circ} \mathrm{C}$ and separated by SDSPAGE and probed by rabbit anti-MAP4 (A301-489A, Bethyl Laboratories) antibodies using western blotting.

Statistical analysis. Data represent means \pm S.E.M. of $n$ experiments. The data meet the assumptions of the tests. Statistical differences between groups were assessed by two-tailed Student's $t$-test or one-way analysis of variance (ANOVA) post hoc tests, as appropriate. In all comparisons, a $P$-value of $<0.05$ was considered statistically significant. Statistical analyses were performed with the software program IBM SPSS STATISTIC, version 19 (SPSS Inc., Chicago, IL, USA) and PASS 11 (NCSS Inc., Williamsport, PA, USA).

\section{Conflict of Interest}

The authors declare no conflict of interest.
Acknowledgements. This study was supported by the National Natural Science Foundation of China (nos. 81055748 and 81270030), National Program on Key Basic Research Project of China (973 Program; no. 2012CB518101) and State Key Laboratory of Trauma, Burns and Combined Injury Research Foundation (nos. SKLZZ200909, 201008, 201224 and 200806). We thank Dr. Xiao-jun Du for comments and suggestions of earlier versions of the paper.

\section{Author contributions}

YS Huang supervised the work; J Hu, J Zhang and Y Huang designed the experiments with help from HC Chan and J Ren; J Hu, Z Chu, Q Zhang, D Zhang, $\mathrm{J} H$ Han and $Y$ Dang conducted the experiments; J Hu and J Han analyzed the data; $\mathrm{J} \mathrm{Hu}$, J Zhang and HC Chan wrote the manuscript.

1. Gersh BJ, Sliwa K, Mayosi BM, Yusuf S. The epidemic of cardiovascular disease in the developing world: global implications. Eur Heart J 2010; 31: 642-648.

2. World Health Organization Global Status Report on Noncommunicable Diseases 2010. World Health Organization: Geneva, 2011.

3. Li Y, Ge S, Peng Y, Chen X. Inflammation and cardiac dysfunction during sepsis, muscular dystrophy, and myocarditis. Burn Trauma 2013; 1: 109-121.

4. Narula J, Haider N, Arbustini E, Chandrashekhar Y. Mechanisms of disease: apoptosis in heart failure-seeing hope in death. Nat Clin Pract Cardiovasc Med 2006; 12: 681-688.

5. Gill C, Mestril R, Samali A. Losing heart: the role of apoptosis in heart disease-a novel therapeutic target? FASEB J 2002; 16: 135-146.

6. Baines CP. The molecular composition of the mitochondrial permeability transition pore. J Mol Cell Cardiol 2009; 46: 850-857.

7. Cory S, Adams JM. The Bcl2 family: regulators of the cellular life-or-death switch. Nat Rev Cancer 2002; 2: 647-656.

8. Cheng Z, Völkers M, Din S, Avitabile D, Khan M, Gude N et al. Mitochondrial translocation of Nur77 mediates cardiomyocyte apoptosis. Eur Heart $J$ 2011; 32: 2179-2188.

9. Miura T, Miki T. GSK-3beta, a therapeutic target for cardiomyocyte protection. Circ J2009; 73: $1184-1192$.

10. Lu G, Haider HKh, Porollo A, Ashraf M. Mitochondria-specific transgenic overexpression of connexin-43 simulates preconditioning-induced cytoprotection of stem cells. Cardiovasc Res 2010; 88: 277-286.

11. Chua BT, Volbracht C, Tan KO, Li R, Yu VC, Li P. Mitochondrial translocation of cofilin is an early step in apoptosis induction. Nat Cell Biol 2003; 12: 1083-1089.

12. Zha J, Harada H, Yang E, Jockel J, Korsmeyer SJ. Serine phosphorylation of death agonist BAD in response to survival factor results in binding to 14-3-3 not BCL-X(L). Cell 1996; 87: 619-628.

13. Illenberger S, Drewes G, Trinczek B, Biernat J, Meyer HE, Olmsted JB et al. Phosphorylation of microtubule-associated proteins MAP2 and MAP4 by the protein kinase p110mark. Phosphorylation sites and regulation of microtubule dynamics. J Biol Chem 1996; 271: 10834-10843.

14. Drewes G, Ebneth A, Mandelkow EM. MAPs, MARKs and microtubule dynamics MAPs. Trends Biochem Sci 1998; 23: 307-311.

15. Ebneth A, Drewes G, Mandelkow EM, Mandelkow E. Phosphorylation of MAP2c and MAP4 by MARK kinases leads to the destabilization of microtubules in cells. Cell Motil Cytoskeleton 1999; 44: 209-224.

16. Hu JY, Chu ZG, Han J, Dang YM, Yan H, Zhang $Q$ et al. The $\mathrm{p} 38 / M A P K$ pathway regulates microtubule polymerization through phosphorylation of MAP4 and Op18 in hypoxic cells. Cell Mol Life Sci 2010; 67: 321-333.

17. Linden $M$, Nelson $B D$, Leterrier JF. The specific binding of the microtubule-associated protein 2 (MAP2) to the outer membrane of rat brain mitochondria. Biochem J 1989; 261: 167-173.

18. Pan JA, Ullman E, Dou Z, Zong WX. Inhibition of protein degradation induces apoptosis through a microtubule-associated protein 1 light chain 3-mediated activation of caspase-8 at intracellular membranes. Mol Cell Biol 2011; 31: 3158-3170.

19. Chen ZH, Lam HC, Jin Y, Kim HP, Cao JF et al. Autophagy protein microtubule-associated protein 1 light chain-3B (LC3B) activates extrinsic apoptosis during cigarette smokeinduced emphysema. Proc Natl Acad Sci USA 2010; 107: 18880-18885.

20. Eckert A, Schulz KL, Rhein V, Gotz J. Convergence of amyloid-beta and tau pathologies on mitochondria in vivo. Mol Neurobiol 2010; 41: 107-114.

21. David DC, Hauptmann S, Scherping I, Schuessel K, Keil U, Rizzu P et al. Proteomic and functional analyses reveal a mitochondrial dysfunction in P301L tau transgenic mice. J Biol Chem 2005; 280: 23802-23814.

22. Wang ZF, Yin J, Zhang Y, Zhu LQ, Tian Q, Wang XC et al. Overexpression of tau proteins antagonizes amyloid-beta-potentiated apoptosis through mitochondria-caspase-3 pathway in N2a cells. J Alzheimers Dis 2010; 20: 145-157.

23. Srsen V, Kitazawa H, Sugita M, Murofushi H, Bulinski JC et al. Serum-dependent phosphorylation of human MAP4 at Ser696 in cultured mammalian cells. Cell Struct Funct 1999; 24: 321-327. 
24. Kitazawa H, lida J, Uchida A, Haino-Fukushima K, Itoh TJ, Hotani H et al. Ser787 in the proline-rich region of human MAP4 is a critical phosphorylation site that reduces its activity to promote tubulin polymerization. Cell Struct Funct 2000; 25: 33-39.

25. Yang $\mathrm{H}$, Ganguly $\mathrm{A}$, Cabral $\mathrm{F}$. Inhibition of cell migration and cell division correlates with distinct effects of microtubule inhibiting drugs. J Biol Chem 2010; 285: 32242-32250.

26. Prokop A. The intricate relationship between microtubules and their associated moto proteins during axon growth and maintenance. Neural Dev 2011; 8: 17.

27. Samora CP, Mogessie B, Conway L, Ross JL, Straube A, McAinsh AD. MAP4 and CLASP1 operate as a safety mechanism to maintain a stable spindle position in mitosis. Nat Cell Biol 2011; 13: 1040-1050.

28. Teng M, Jiang XP, Zhang Q, Zhang JP, Zhang DX, Liang GP et al. Microtubular stability affects $\mathrm{pVHL}$-mediated regulation of HIF-1alpha via the p38/MAPK pathway in hypoxic cardiomyocytes. PLoS One 2012; 7: e35017.

29. Teng M, Dang YM, Zhang JP, Zhang Q, Fang YD, Ren J et al. Microtubular stability affects cardiomyocyte glycolysis by HIF-1alpha expression and endonuclear aggregation during early stages of hypoxia. Am J Physiol Heart Circ Physiol 2010; 298: H1919-H1931.

30. Tomimoto $\mathrm{H}$, Yanagihara $\mathrm{T}$. Immunoelectron microscopic study of tubulin and microtubuleassociated proteins after transient cerebral ischemia in gerbils. Acta Neuropathol 1992; 84 394-399.

31. Marzo I, Brenner C, Zamzami N, Jürgensmeier JM, Susin SA, Vieira HL et al. Bax and adenine nucleotide translocator cooperate in the mitochondrial control of apoptosis. Science 1998; 281: 2027-2031.
32. Bottero V, Rossi F, Samson M, Mari M, Hofman P, Peyron JF. Ikappa b-alpha, the NF-kappa B inhibitory subunit, interacts with ANT, the mitochondrial ATP/ADP translocator. J Biol Chem 2001; 276: 21317-21324.

33. Jiang XS, Dai J, Sheng QH, Zhang L, Xia QC, Wu JR et al. A comparative proteomic strategy for subcellular proteome research: ICAT approach coupled with bioinformatics prediction to ascertain rat liver mitochondrial proteins and indication of mitochondrial localization for catalase. Mol Cell Proteomics 2005; 4: 12-34.

Cell Death and Disease is an open-access journal published by Nature Publishing Group. This work is licensed under a Creative Commons Attribution-NonCommercialShareAlike 3.0 Unported License. The images or other third party material in this article are included in the article's Creative Commons license, unless indicated otherwise in the credit line; if the material is not included under the Creative Commons license, users will need to obtain permission from the license holder to reproduce the material. To view a copy of this license, visit http://creativecommons.org/licenses/ by-nc-sa/3.0/

Supplementary Information accompanies this paper on Cell Death and Disease website (http://www.nature.com/cddis) 